\title{
ORIGINAL
}

\section{IMPACTO DEL REAL DECRETO-LEY 16/2012 SOBRE EL COPAGO FARMACEUTICO EN EL NÚMERO DE RECETAS Y EN EL GASTO FARMACÉUTICO}

\author{
Fernando Antoñanzas Villar, Roberto Rodríguez-Ibeas, Carmelo A Juárez-Castelló y $\mathbf{M}^{\mathrm{a}}$ Reyes \\ Lorente Antoñanzas.
}

Departamento de Economía y Empresa. Universidad de La Rioja.

\begin{abstract}
RESUMEN
Fundamentos: el objetivo del trabajo es es conocer si el impacto del Real Decreto-Ley 16/2012 en el número de recetas y el gasto farmacéutico, evaluadas por el Ministerio de Sanidad, Servicios Sociales e Igualdad (MSSSI), se corresponden con las obtenidas por otros métodos estadísticos habitualmente empleados. Asimismo, se han elaborado unos modelos para predecir la evolución de ambas variables entre septiembre de 2013 y diciembre de 2014.

Métodos: se aplicó la metodología Box-Jenkins conjuntamente con el análisis de intervención de Box-Tiao a datos del periodo 2003-13 para predecir mensualmente los valores de las series de recetas y gasto farmacéutico. Las predicciones se emplearon en un análisis contrafáctico para compararlas con las series de recetas y gasto real. También se efectuaron predicciones para el periodo de septiembre de 2013 a diciembre de 2014 para observar el impacto de la medida en un horizonte superior al real

Resultados: el análisis contrafáctico estimó el descenso en el número de recetas en un $12,18 \%$ y el del gasto farmaceutico en un $12,83 \%$, mientras que al calcularse mediante el análisis de intervención fueron $12,75 \%$ y $14,03 \%$, respectivamente.
\end{abstract}

Conclusiones: la reducción estimada del número de recetas para el periodo de junio de 2012 hasta agosto de 2013 es similar a la ofrecida por el MSSSI, mientras que para la serie del gasto farmacéutico fue inferior a la ofrecida por el MSSSI. La metodología de Box-Jenkins genera errores de predicción menores al $3 \%$ por lo que se considera útil para anticipar fiablemente los consumos futuros.

Palabras clave: Gasto sanitario. Prescripciones farmacéuticas. Análisis de series temporales. Reforma sanitaria.

\footnotetext{
Correspondencia

Fernando Antoñanzas Villar

Departamento de Economía y Empresa

Universidad de La Rioja

Cigüeña 60

26005-Logroño

España

fernando.antonanzas@unirioja.es
}

\section{ABSTRACT}

Impact of the Royal Decree-Law 16/2012 on the Number of Prescriptions and Pharmaceutical Expenditures

Background: this research aims to understand if the consequences on drug expenditures and number of prescriptions of Royal Decree-Law 16/2012 as estimated by the Ministry of Health, Social Services and Equality (MHSSE) are similar to those found by using common statistical approaches. In addition, several models have been built to forecast the evolution of both variables for the period September 2013-December 2014.

Methods: the Box-Jenkins methodology and the Box-Tiao intervention analysis were applied to data of the period 2003-13 to forecast the monthly values of the number of prescriptions and pharmaceutical expenditures. Forecasts were used in a counter-factual analysis to be compared to the actual values of prescriptions and drug expenditures. Moreover, forecasts for the period September 2013 to December 2014 were obtained to observe the impact of the policy in the future.

Results: the counterfactual analysis estimated a decrease in the number of prescriptions of $12.18 \%$ and $12.83 \%$ in the pharmaceutical expenditure; these figures were $12,75 \%$ and $14,03 \%$ respectively, when the intervention analysis was used.

Conclusion: the estimated reduction in the number of prescriptions for the period June 2012-August 2013 was similar to the figure offered by the MHSSE, while the reduction in the drug expenditure series was smaller. The Box-Jenkins methodology generated low forecast errors (less than $3 \%$ ) what makes this procedure useful to reliably anticipate future consumptions.

Keyword: Health expenditure. Drug prescriptions. Time series analysis. Health care reform. 


\section{INTRODUCCIÓN}

El continuo crecimiento del gasto farmacéutico es una preocupación constante de las autoridades sanitarias de los países desarrollados. España no ha sido ajena a esa cuestión y en las últimas décadas se ha adoptado una diversidad de medidas orientadas a moderar ese crecimiento. Sin embargo, al igual que ha sucedido en el resto de Europa, no existen demasiados estudios que hayan evaluado el impacto de este tipo de medidas de control de costes farmacéuticos, como han señalado Guillen y Cabiedes ${ }^{1}$.

Las principales medidas utilizadas en estas últimas décadas han sido básicamente de cuatro clases: el sistema de precios de referencia, las medidas de ajuste directo del precio de laboratorio (PVL) y de ajuste de los márgenes de distribución, tanto mayorista como de dispensación en oficinas de farmacia, las listas negativas de medicamentos, con exclusión de fármacos previamente incluidos en la financiación pública y la modificación del sistema de copagos (tabla 1). Además, desde el ámbito de las comunidades autónomas, como responsables de la gestión sanitaria, se han adoptado en cada una diferentes tipos de medidas tanto directas (Cataluña y Madrid tuvieron establecido durante un corto periodo de tiempo un copago de $1 €$ por receta en 2012 y 2013) como indirectas orientadas a influir en la prescripción ${ }^{2}$. La grave crisis económica de los últimos años llevó al gobierno a adoptar medidas de intencionalidad más radical y potente orientadas no sólo a frenar el crecimiento del gasto sino incluso a reducirlo.

En este sentido, el Real Decreto-ley $16 / 2012^{3}$ (RDL) se justifica en la aplicación del "principio de austeridad y de racionalización en el gasto público en la oferta de medicamentos" y amplía las variables objeto de regulación. De esta suerte, incluye medidas fundamentalmen- te orientadas a reducir el número de recetas junto con el importe del gasto farmacéutico total. Las medidas de esa norma forman parte de los dos últimos grupos antes descritos: la exclusión de determinados medicamentos de la lista de los financiados públicamente y la implantación del nuevo sistema de copago con el efecto disuasorio del consumo que incorpora. En su motivación económica no se presentaron las cifras esperadas de reducción del número de recetas ni del gasto, pero sí se han ido indicando posteriormente a través de las notas de prensa del propio Ministerio de Sanidad, Servicios Sociales e Igualdad (MSSSI). Así, en octubre de 2013, el importe del ahorro se estimó por el MSSSI $^{4,5}$ en 2.125 millones de $€$ en los 14 meses desde la aplicación de la norma -un $15,58 \%$ - y el descenso en el número de recetas en 127.226.202 (13,07\%).

Como consecuencia de este RDL, con efectos de 1 de septiembre de 2012, la Resolución del MSSSI de 2 de agosto de 2012 excluyó de la financiación pública más de 400 medicamentos, dado que convivían con otros con los que compartían principio activo y dosis o que estaban indicados en el tratamiento de síntomas menores.

El RDL introdujo un cambio sustancial en el sistema de participación del usuario en el coste de la prestación farmacéutica, de manera que vinculó los niveles de renta como criterio relevante para la fijación de los porcentajes de copago (antes general del $40 \%$, y ahora variables: $40 \%, 50 \%$ y $60 \%$ ), estableció un copago del $10 \%$ para los pensionistas (antes exentos) con importes máximos mensuales según sus niveles de renta y fijó que el nivel máximo de aportación reducida por envase se actualizase anualmente según el Índice de Precios al Consumo (4,20€ para 2013).

Transcurrido más de un año desde la aplicación del RDL, se dispone de datos suficientes para evaluar su repercusión en 
Tabla 1

Resumen de medidas aplicadas en el periodo de análisis

\begin{tabular}{|c|c|c|c|c|c|}
\hline Medida & Fecha de la norma & PR & PV & EX & $\mathrm{CO}$ \\
\hline Ley $16 / 2003$ de cohesión y calidad del Sistema Nacional de Salud & Mayo 2003 & $\mathrm{X}$ & & & \\
\hline Orden SCO/2958/2003. Revisión anual sistema de precios de referencia & Octubre 2003 & $\mathrm{X}$ & & & \\
\hline Orden SCO/3524/2003. Modifica la Orden SCO/2958/2003 & Diciembre 2003 & $\mathrm{X}$ & & & \\
\hline Orden SCO/1344/2004. Revisión anual sistema de precios de referencia & Mayo 2004 & $\mathrm{X}$ & & & \\
\hline Real Decreto 2402/2004. Revisiones coyunturales de precios y medidas de contención del gasto farmacéutico. & Diciembre 2004 & & $\mathrm{X}$ & & \\
\hline Ley $29 / 2006$ de garantías y uso racional de los medicamentos y productos sanitarios & Julio 2006 & $\mathrm{X}$ & & & \\
\hline Real Decreto 1338/2006. Desarrolla la Ley 29/2006 & Noviembre 006 & $\mathrm{X}$ & & & \\
\hline Orden SCO/3997/2006. Revisión anual y desarrollo Ley 29/2006 & Diciembre 2006 & $\mathrm{X}$ & & & \\
\hline Orden SCO/3867/2007. Revisión anual sistema precios de referencia & Diciembre 2007 & $\mathrm{X}$ & & & \\
\hline Real Decreto 823/2008. Márgenes, deducciones y descuentos en la distribución & Mayo 2008 & & $\mathrm{X}$ & & \\
\hline Orden SCO/3803/2008. Revisión anual sistema precios de referencia & Diciembre 2008 & $\mathrm{X}$ & & & \\
\hline Orden SAS/3499/2009. Revisión anual sistema precios de referencia & Diciembre 009 & $\mathrm{X}$ & & & \\
\hline RDL 4/2010, de racionalización del gasto farmacéutico con cargo al Sistema Nacional de Salud. & Marzo 2010 & & $\mathrm{X}$ & & \\
\hline $\begin{array}{l}\text { RDL } 8 / 2010 \text {, por el que se adoptan medidas extraordinarias para } \\
\text { la reducción del déficit público. }\end{array}$ & Mayo 2010 & & $\mathrm{X}$ & & \\
\hline Orden SPI/3052/2010. Revisión anual sistema precios de referencia & Noviembre 010 & $\mathrm{X}$ & & & \\
\hline RDL 9/2011 de medidas para la mejora de la calidad y cohesión del Sistema Nacional de Salud & Agosto 2011 & $\mathrm{X}$ & $\mathrm{X}$ & & \\
\hline Resolución de actualización Orden SPI/3052/2010 & Diciembre 2011 & $\mathrm{X}$ & & & \\
\hline RDL 16/2012 modificado por RDL 19/2012 - Ley 12/2012 & Abril 2012 & $\mathrm{X}$ & & $\mathrm{X}$ & $\mathrm{X}$ \\
\hline Resolución de actualización aportación reducida & Mayo 2012 & & & & $\mathrm{X}$ \\
\hline Resolución de lista exclusión de la prestación & Agosto 2012 & & & $\mathrm{X}$ & \\
\hline Resolución de actualización aportación reducida & Enero 2013 & & & & $\mathrm{X}$ \\
\hline
\end{tabular}

PR: Precios de referencia; PV:Ajustes de precios y márgenes de distribución; EX:listas negativas; CO:copagos.

No se incluyen algunas normativas relacionadas con el copago en la dispensación hospitalaria dado que no afectan a la serie objeto de estudio. 
las variables cuya evolución pretendía modificar. El análisis de las consecuencias reales de las políticas públicas en general es una tarea compleja, que no siempre se ha llegado a abordar debido, quizás, a la carencia de datos, a la falta de voluntad política para evaluar los resultados o a la dificultad inherente en los métodos para dicho análisis.

En el caso que nos ocupa, los cambios derivados del RDL se cuantificaron mensualmente por el MSSSI ${ }^{4,5}$ y por otras instituciones, midiéndose con respecto al mes precedente, con respecto a los doce meses precedentes a la entrada en vigor de la nueva normativa ( 1 de julio de 2012 para todo el Estado, excepto para el País Vasco que aplicó la medida con un año de retraso) o bien con respecto al mismo mes en el año anterior para suavizar las distorsiones de las variaciones estacionales. Sin embargo, cuando se analizó mediante las mencionadas comparaciones el valor del número de recetas y del gasto farmacéutico en los meses de julio y agosto de 2013 se detectó un repunte en el consumo. Ese hallazgo se creyó debido a que la base de la comparación eran los datos de julio y agosto de 2012, anormalmente menores, puesto que hubo un efecto de acumulación de recetas durante junio de 2012 por parte de algunos pacientes para protegerse algo del impacto posterior de la medida. Dicho de otro modo, los pacientes precisaron menos recetas en julio y agosto de 2012 ya que las habían obtenido en junio. De esta forma, los resultados del consumo en julio y agosto de 2013 apuntaban a un desgaste de la capacidad de la política aplicada para logar los menores consumos perseguidos puesto que los valores habían crecido sustancialmente. En este contexto, se pasó, por parte de las autoridades sanitarias (véanse las diferencias en las notas de prensa del MSSSI $^{4,5}$ ) a establecer la base de la comparación con lo acontecido dos años antes, es decir, durante los mismos meses del año 2011. Esto es, ha habido un cambio en la forma de presentación de algunos de los resultados de la influencia del nuevo RDL.

Ante esta situación de falta de un criterio claro y homogéneo vale la pena intentar examinar de una forma más sofisticada en términos estadísticos el cambio acontecido en la serie de recetas y de gasto tras la aplicación de las medidas de política farmacéutica incluidas en el citado RDL. Para ello existe un método, ya acuñado en la literatura, que es el análisis mediante los modelos ARIMA de Box-Jenkins ${ }^{6}$, ya utilizado por otros autores con similares propósitos (Moreno ${ }^{2}, \mathrm{Lee}^{7}, \mathrm{Marshall}^{8}$ Ong ${ }^{9}$ y Pichetti $\left.^{10}\right)$.

El objetivo del presente trabajo fue conocer si las repercusiones del RDL en cuanto al descenso en el consumo de fármacos (número de recetas y valor del gasto farmacéutico) evaluado por el MSSSI se corresponde con las obtenidas por los métodos estadísticos más sofisticados, habitualmente empleados para esta clase de mediciones. El segundo objetivo fue elaborar unos modelos para predecir la evolución de ambas variables durante el periodo comprendido entre los meses de septiembre de 2013 y diciembre de 2014

\section{MATERIAL Y MÉTODOS}

Para cuantificar las repercusiones de las medidas se utilizaron los modelos ARIMA ${ }^{6}$ junto con las técnicas del análisis de intervención de Box y Tiao ${ }^{11}$. Mediante estos instrumentos se captó la estructura dinámica de la serie de datos, se cuantificó el potencial cambio registrado en la serie ante influencias externas como una nueva regulación administrativa y se pueden efectuar predicciones a corto plazo con un mínimo de información disponible (el propio pasado de la serie) con reducidos niveles de error que son calificadas de óptimas en términos de su reducida varianza. 
Los modelos ARIMA requieren disponer de una serie larga de datos si se trata de observaciones mensuales, para captar los componentes estacional y cíclico que pudieran estar presentes en la variable. De este modo, en nuestro estudio se dispuso de los datos de gasto farmacéutico y del número de recetas que publica el MSSSI en su página web $^{12}$ desde enero 2003 hasta agosto de 2013. El software para el tratamiento estadístico de los datos fue Eviews ${ }^{6}$.

Para analizar los efectos de las nuevas medidas de contención del gasto público en medicamentos del RDL se llevaron a cabo dos clases de análisis. En primer lugar, se elaboró un escenario contrafáctico del tipo: ¿qué hubiera ocurrido con la evolución de las variables del gasto farmacéutico y del número de recetas en ausencia del RDL. Por diferencia entre los valores reales y los de tal escenario, se cuantificó el efecto atribuible a la medida tanto en valores absolutos como relativos (porcentajes). Para llevar a cabo este análisis contrafáctico se empleó la metodología de Box-Jenkins ${ }^{6}$, identificando, estimando y verificando los modelos ARIMA de cada una de las series objeto de estudio. La muestra de observaciones comprendió el período de enero de 2003 a mayo de 2012 (justo un mes antes de la aplicación de las medidas de contención del gasto del citado RDL, uno de julio de 2012). A partir de las estimaciones de los mejores modelos que explicaron la evolución de ambas series se calcularon las predicciones generadas para el período junio de 2012 hasta agosto de 2013. Estas predicciones habrían marcado la evolución de la serie en ausencia del RDL (y, por extensión, de cualquier otro cambio en el periodo). Por comparación, restando de las predicciones de recetas realmente dispensadas y de sus correspondientes valores de gasto las cifras reales consumidas, se dispuso de los importes resultantes, los denominados ahorros logrados mediante la medida. El resultado de la diferencia generó valores en términos absolutos. Para calcular los ahorros en tér- minos relativos respecto de lo que hubiese acontecido en ausencia del RDL se utilizó la fórmula:

Valores reales-valores predichos/valores predichos

En segundo lugar, para corroborar los resultados anteriores se realizó un análisis de intervención (Box y Tiao $^{11}$ ) que usa variables ficticias para captar el impacto en las series de recetas y de gasto farmacéutico de las diferentes medidas legislativas adoptadas. Las variables ficticias se definieron con valores cero o uno desde enero de 2003 hasta agosto de 2013 cuando existía un fenómeno determinista ajeno a la propia dinámica aleatoria de las series que afectaba a su evolución. En el caso de las series estudiadas esos fenómenos habrían sido los cambios normativos mencionados en la introducción. No obstante, a la vista de los correlogramas y de los análisis gráficos de la serie y de los residuos de las estimaciones iniciales, entre todos ellos se eligieron los momentos en que los efectos de las medidas o de otras circunstancias de origen incierto -variables ficticias introducidas de forma empírica sin conocer con precisión la naturaleza del fenómeno que motivó la alteración en los valores de la serie- parecían haber afectado más su evolución. Estas variables se diseñaron como "impulsos" o "escalones", según la terminología al uso, dependiendo de que respectivamente efectos transitorios o permanentes en el nivel de la serie. En particular, se definieron las siguientes variables ficticias: 1) la variable FJUN12, que tomó el valor -1 para el mes de junio de 2012, el valor 1 para los meses de julio 2012 a agosto 2013 y el valor 0 para el resto de los períodos. Esta variable captaría el efecto acumulación de junio de 2012 así como el impacto del RDL a partir del mes de julio de 2012;2) la variable FENE10 tomó el valor 1 para los meses de enero y febrero de 2010 y el valor 0 para el resto de meses; 3 ) la variable FNOV11 que tomó el valor 1 para el mes de noviembre de 2011 y el valor 0 para el resto de los meses; 4) la variable 
DJUN12 que tomó el valor el valor 1 para los meses junio 2012-agosto 2013 y el valor 0 para el resto de los periodos; 5) la variable FJUN10 que tomó el valor 1 para los meses de junio y julio de 2010 y el valor 0 en el resto de los meses. De las variables FJUN12 y DJUN12 se tomaron primeras diferencias estacionales y no estacionales.

Al estimar los parámetros de las variables ficticias mediante el análisis de intervención, se incorporó la propia estructura dinámica de las series, es decir, sus componentes tendenciales, cíclicos y estacionales si los hubo. De esta manera, se identificó o aisló el efecto de la medida de regulación de la evolución natural de la variable, aunque no se conociera si otras causas o variables también influyeron en los cambios al tratarse de un análisis de carácter univariante.

Por otra parte, relacionado con el segundo de los objetivos de este estudio, se ha de señalar que estos análisis de series temporales permiten "controlar" la evolución del fenómeno (en la terminología de Box-Jenkins $^{6}$ ), esto es, facilitan averiguar si en un momento dado la evolución de la variable cambia del patrón esperado. Para ello fue preciso efectuar predicciones y cotejarlas con los datos reales disponibles a medida que fueron estando disponibles. Así se contrastó si el cambio promovido por la política continuó o no surtiendo el efecto perseguido.

Para tal fin, tras haber estimado los parámetros de los modelos ARIMA y haber comprobado la calidad estadística de las estimaciones en la fase de verificación se pasó a efectuar las predicciones. Se hicieron hasta el horizonte de diciembre de 2014 para los modelos estimados con los datos desde enero de 2003 hasta agosto de 2013 (los correspondientes al análisis de intervención). Las predicciones se efectuaron con sus correspondientes intervalos de confianza (al nivel de confianza del 80\%, comúnmente aplicado por suponer un compromiso aceptable entre precisión y amplitud del intervalo). De esta suerte, se dispuso de ese conjunto de valores para, cuando mensualmente se conozcan los valores reales, continuar calculando las repercusiones de la medida y comprobar si se mantiene la reducción en los niveles de las variables hasta ese citado horizonte de diciembre de 2014.

\section{RESULTADOS}

Tras haber aplicado la secuencia indicada en la metodología de identificación-estimación-verificación a varias identificaciones iniciales, en el análisis contrafáctico del número de recetas el modelo que mejor superó la fase de verificación para la serie en logaritmos fue ARIMA $(2,1,3)(0,1,1) 12$, quedando restringidos a cero los coeficientes de órdenes 1 y 2 del término de medias móviles no estacionales e incluyendo las variables ficticias FNOV11 y FENE10 para captar los efectos de las medidas legislativas de los años 2010 y 2011. Todos los coeficientes fueron significativos al nivel del $1 \%$. Los resultados de este modelo se presentan en la tabla 2. Cabe destacar que el error estándar de la estimación del modelo es de $2,3 \%$. Los coeficientes de las variables ficticias fueron $-0,0246 \mathrm{y}$ 0,0105 , indicando que el descenso atribuible a las medidas legislativas fue de 2,42\% [calculado como exp(-0,0246)-1)] y $1,04 \%$, respectivamente.

El modelo se utilizó para predecir el número de recetas para el periodo junio 2012-agosto 2013. Las predicciones se muestran en la tabla 3 . El número de recetas predichas por el modelo para cada período fue mayor (en un intervalo de entre 6 y 17 millones según los meses) que su volumen real (excepto para el mes de junio de 2012), ya que el modelo no recogió el efecto del RDL. Por tanto, el efecto fue disminuir el número de recetas durante el período considerado en un 12,18 \% (aplicando la fórmula descrita en la sección de Material y Métodos). En la figura 1 se muestran las predic- 
Tabla 2

Modelos estimados para la serie del número de recetas

\begin{tabular}{|l|c|c|c|c|}
\hline \multicolumn{2}{|c|}{ Variable } & \multicolumn{2}{c|}{$\begin{array}{c}\text { Análisis contrafáctico } \\
\text { (Enero 2003-Mayo 2012) }\end{array}$} & \multicolumn{2}{c|}{$\begin{array}{c}\text { Análisis de intervención } \\
\text { (Enero 2003-Agosto 2013) }\end{array}$} \\
\hline & Coeficiente & Error estándar & Coeficiente & Error estándar \\
\hline FNOV11 & $-0,024601$ & 0,007661 & $-0,030491$ & 0,009622 \\
\hline FENE10 & $-0,010552$ & 0,002901 & $-0,010386$ & 0,002917 \\
\hline FJUN12 & & & $-0,136413$ & 0,010052 \\
\hline Término autorregresivo 1 & $-1,106465$ & 0,027363 & $-1,145320$ & 0,026272 \\
\hline Término autorregresivo 2 & $-0,965917$ & 0,030960 & $-0,973486$ & 0,027325 \\
\hline Término de medias móviles estacional 1 & $-0,883982$ & 0,029065 & $-0,775507$ & 0,062867 \\
\hline Termino de medias móviles MA(3) & $-0,810453$ & 0,075501 & $-0,867091$ & 0,059480 \\
\hline Observaciones & 98 & & 113 & \\
\hline Q test (p-valor, retardo 36) & $23,791(0,852)$ & & $19,817(0,954)$ & \\
\hline Suma de cuadrados de los residuos & 0,051854 & & 0,079731 & \\
\hline Error estándar de la regresión & 0,023741 & & 0,027426 & \\
\hline Modelo ARIMA & $(2,1,3)(0,1,1) 12$ & & $(2,1,3)(0,1,1) 12$ & \\
\hline
\end{tabular}

ciones puntuales para la serie de recetas, así como sus correspondientes intervalos de confianza al $80 \%$ de nivel de confianza. Todos los valores reales de la serie número de recetas quedaron fuera de estos intervalos, lo cual indicó que hubo un cambio en la estructura de la serie como consecuencia del RDL.

\section{Tabla 3}

\section{Predicciones del análisis contrafáctico para el número de recetas del periodo Junio} 2012-Agosto 2013

\begin{tabular}{|l|c|c|c|c|}
\hline & $\begin{array}{c}\text { Número de recetas } \\
\text { reales }\end{array}$ & $\begin{array}{c}\text { Número } \\
\text { derecetas } \\
\text { predichas }\end{array}$ & Intervalo de confianza 80\% & $\begin{array}{c}\text { Recetas predichas } \\
\text { menos } \\
\text { recetas reales }\end{array}$ \\
\hline JUN 2012 & 90.031 .681 & 81.663 .940 & $79.716 .112-84.181 .653$ & -8.367 .741 \\
\hline JUL 2012 & 67.439 .043 & 78.826 .810 & $76.428 .993-81.253 .388$ & 11.387 .767 \\
\hline AG 2012 & 69.838 .933 & 76.436 .721 & $74.087 .812-78.814 .097$ & 6.597 .788 \\
\hline SEPT 2012 & 62.799 .047 & 79.977 .826 & $77.484 .499-82.501 .812$ & 17.178 .779 \\
\hline OCT 2012 & 72.313 .243 & 83.369 .264 & $80.773 .973-85.996 .421$ & 11.056 .021 \\
\hline NOV 2012 & 67.721 .707 & 82.360 .419 & $79.756 .716-84.996 .594$ & 14.638 .712 \\
\hline DIC 2012 & 66.433 .909 & 81.470 .890 & $78.876 .356-84.098 .025$ & 15.036 .981 \\
\hline EN 2013 & 73.940 .734 & 84.449 .410 & $81.763 .525-87.168 .998$ & 10.508 .676 \\
\hline FEBR 2013 & 68.149 .249 & 82.461 .690 & $79.790 .932-85.166 .583$ & 14.312 .441 \\
\hline MARZ 2013 & 69.391 .206 & 83.086 .657 & $80.389 .354-85.818 .517$ & 13.695 .451 \\
\hline ABR 2013 & 75.741 .934 & 81.814 .677 & $79.162 .087-84.501 .206$ & 6.072 .743 \\
\hline MAY 2013 & 75.429 .932 & 84.871 .477 & $82.064 .601-87.714 .996$ & 9.441 .545 \\
\hline JUN2013 & 70.376 .601 & 81.048 .333 & $78.303 .627-83.829 .740$ & 10.671 .732 \\
\hline JUL 2013 & 73.799 .890 & 79.926 .033 & $77.260 .308-82.626 .857$ & 6.126 .143 \\
\hline AG 2013 & 66.265 .771 & 76.368 .347 & $73.768 .197-79.003 .455$ & 10.102 .576 \\
\hline TOTAL & 1.069 .672 .880 & 1.218 .132 .494 & & 148.459 .614 \\
\hline
\end{tabular}




\section{Figura 1}

Análisis contrafáctico de la serie de recetas

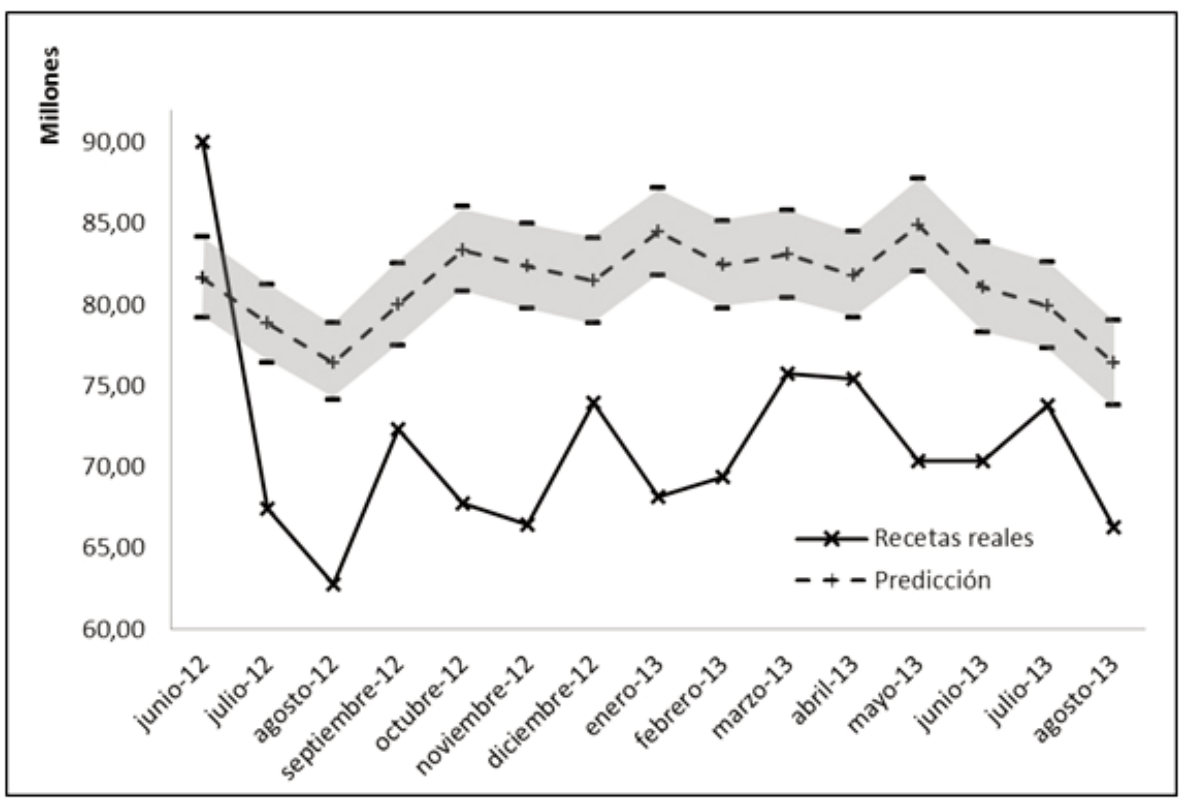

En el análisis de intervención, se estimó el modelo ARIMA $(2,1,3)(0,1,1) 12$ que incluyó las variables FJUN12, FNOV11 y FENE10. Todos los coeficientes estimados fueron estadísticamente significativos al nivel de significación del $1 \%$. Los resultados de la estimación se muestran en la tabla 2. El coeficiente de la variable FJUN12 tomó el valor -0,136413, interpretándose por consiguiente que el número de recetas disminuyó un 12,75\% como consecuencia del RDL. El error estándar del modelo fue de $2,7 \%$. Los coeficientes estimados de las restantes variables ficticias apenas mostraron diferencias con los obtenidos en el análisis contrafáctico.

Las predicciones realizadas con este modelo para el periodo de septiembre de 2013 a diciembre de 2014 se muestran en la tabla 4 y en el figura 2. En ambas se aprecia una estabilización en la evolución de la serie durante los siguientes 14 meses en el entorno de los 70 millones de recetas mensuales, tras el brusco descenso acontecido con motivo del RDL.
En la figura 3 se muestran los residuos del modelo del número de recetas estimado con análisis de intervención.

En el análisis contrafáctico del gasto farmacéutico, tras haber aplicado también la secuencia indicada en la metodología de identificación-estimación-verificación a varias identificaciones iniciales, el modelo que mejor superó la fase de verificación para la serie en logaritmos fue ARIMA $(2,1,0)(0,0,2) 12$. Este modelo exigió la inclusión de un coeficiente de medias móviles para el retardo 10 (introducido en el modelo de forma empírica, a la vista de los correlogramas en las fases de verificación de estimaciones preliminares) así como las variables ficticias FNOV11 y FJUN10, que recogieron el efecto de las medidas legislativas de los años 2010 y 2011. Todos los coeficientes fueron significativos al $1 \%$. Los resultados de este modelo se muestran en la tabla 5. El error estándar fue de 2,4\% y los coeficientes de las variables ficticias fueron de $-0,058 \mathrm{y}-$ 
Tabla 4

Predicciones del análisis de intervención para el número de recetas y el gasto farmacéutico junto con los intervalos de confianza al $80 \%$ para el período de septiembre de 2013 a diciembre de 2014

\begin{tabular}{|l|c|c|c|c|}
\hline \multicolumn{1}{|c|}{ MES } & Recetas predichas & Intervalo de confianza 80\% & $\begin{array}{c}\text { Gasto } \\
\text { predicho }\end{array}$ & Intervalo de confianza 80\% \\
\hline SEPT 2013 & 67.298 .983 & $64.395 .330-70.252 .283$ & 722.422 .892 & $694.010 .446-751.277 .513$ \\
\hline OCT 2013 & 75.427 .368 & $72.721 .981-78.171 .099$ & 815.918 .374 & $783.642 .405-848.699 .608$ \\
\hline NOV 2013 & 67.183 .471 & $64.702 .654-69700.502$ & 714.281 .285 & $684.304 .805-744.756 .091$ \\
\hline DIC 2013 & 69.676 .418 & $66.679 .453-72.724 .466$ & 764.485 .936 & $727.370 .823-802.316 .670$ \\
\hline EN 2014 & 75.563 .003 & $72.760 .410-78.406 .693$ & 792.350 .106 & $754.062 .604-831.372 .305$ \\
\hline FEBR 2014 & 67.104 .575 & $64.498 .105-69.751 .098$ & 722.532 .620 & $685.865 .222-759.939 .676$ \\
\hline MAR 2014 & 72.667 .747 & $69.587 .992-75.799 .212$ & 789.818 .794 & $748.288 .157-832.218 .099$ \\
\hline ABR 2014 & 73.443 .998 & $70.628 .252-76.302 .445$ & 823.604 .241 & $779.890 .017-868.241 .581$ \\
\hline MAY 2014 & 70.403 .220 & $67.505 .775-73.347 .884$ & 805.536 .340 & $760.741 .366-851.323 .374$ \\
\hline JUN 2014 & 71.503 .861 & $68.515 .867-74.541 .311$ & 790.843 .559 & $746.869 .767-835.971 .133$ \\
\hline JUL 2014 & 70.609 .991 & $67.833 .512-73.429 .670$ & 823.521 .529 & $777.443 .176-870.626 .817$ \\
\hline AGO 2014 & 63.848 .213 & $61.097 .454-66.645 .936$ & 752.206 .859 & $709.086 .001-796.312 .858$ \\
\hline SEP 2014 & 69.431 .784 & $65.643 .586-73.302 .261$ & 765.825 .727 & $711.188 .478-822.025 .147$ \\
\hline OCT 2014 & 73.347 .868 & $70.294 .243-76.451 .844$ & 837.347 .910 & $776.359 .641-900.117 .432$ \\
\hline NOV 2014 & 66.734 .149 & $63.495 .681-70.035 .030$ & 779.752 .018 & $720.353 .175-840.967 .702$ \\
\hline DIC 2014 & 71.546 .067 & $67.851 .966-75.316 .011$ & 782.100 .872 & $718.385 .749-847.904 .603$ \\
\hline
\end{tabular}

Figura 2

Serie de recetas con predicciones

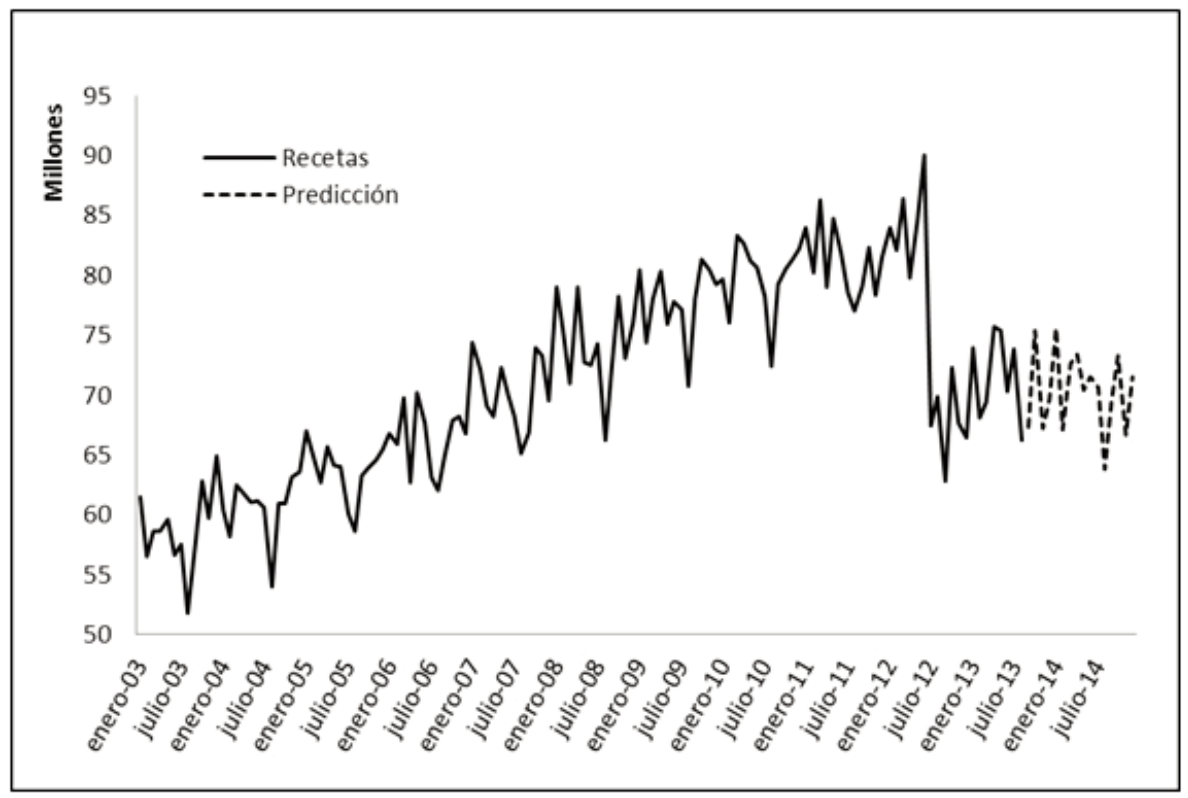


Figura 3

Residuos del modelo de la serie de recetas con análisis de intervención

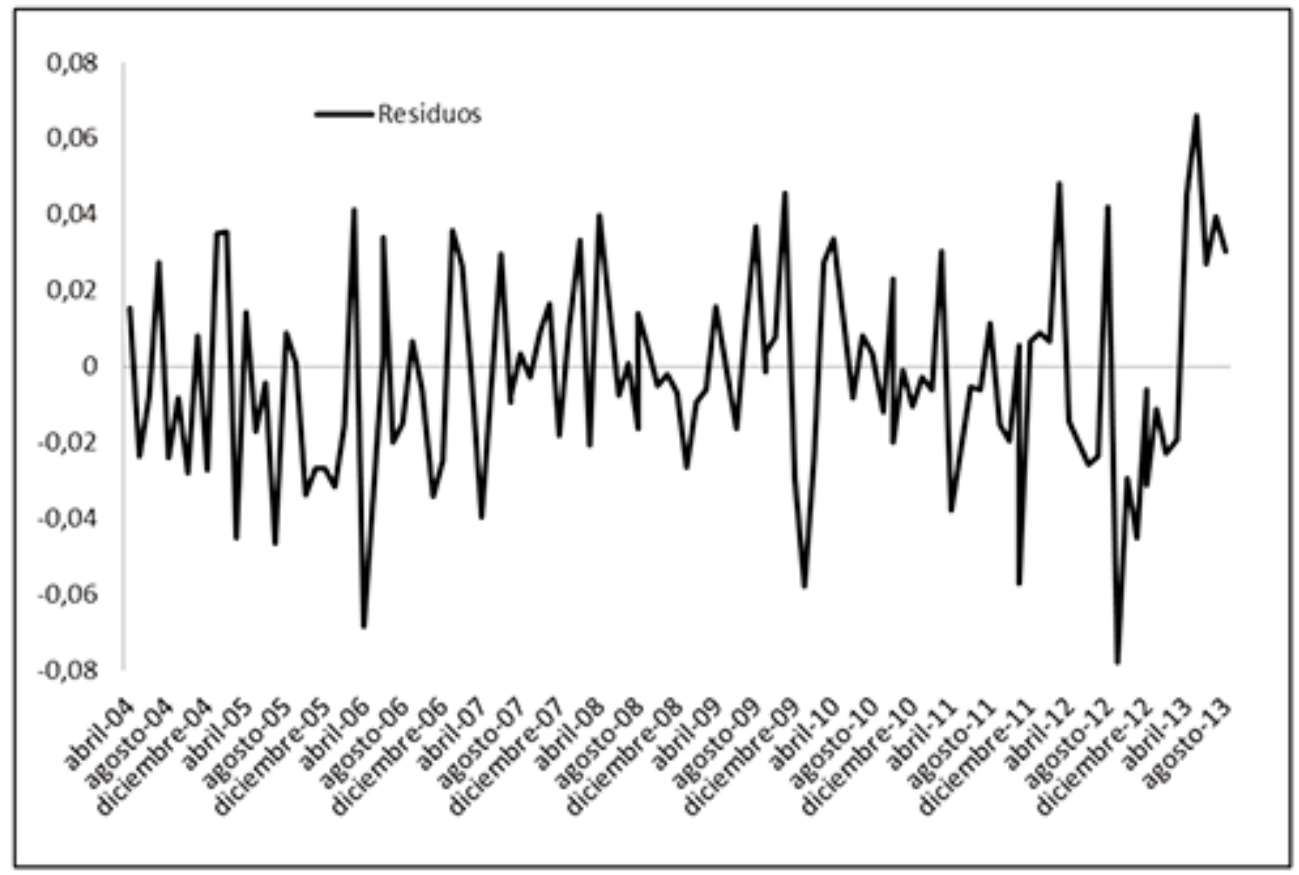

Tabla 5

Modelos estimados para la serie de gasto farmacéutico

\begin{tabular}{|c|c|c|c|c|}
\hline \multirow{2}{*}{ Variable } & \multicolumn{2}{|c|}{$\begin{array}{c}\text { Análisis contrafáctico } \\
\text { (Enero 2003-Mayo 2012) }\end{array}$} & \multicolumn{2}{|c|}{$\begin{array}{c}\text { Análisis de intervención } \\
\text { (Enero 2003-Agosto 2013) }\end{array}$} \\
\hline & Coeficiente & $\begin{array}{c}\text { Error } \\
\text { estándar }\end{array}$ & Coeficiente & $\begin{array}{c}\text { Error } \\
\text { E stándar }\end{array}$ \\
\hline FNOV11 & $-0,058195$ & 0,013185 & $-0,070520$ & 0,016005 \\
\hline FJUN10 & $-0,056085$ & 0,007357 & $-0,050369$ & 0,008830 \\
\hline DFJUN12 & & & $-0,151215$ & 0,010457 \\
\hline Término autorregresivo 1 & $-0,932072$ & 0,073074 & $-0,817604$ & 0,082552 \\
\hline Término autorregresivo 2 & $-0,544935$ & 0,076773 & $-0,508001$ & 0,081451 \\
\hline Término de medias móviles stacional 1 & 0,923970 & 0,000529 & 0,897990 & 0,038552 \\
\hline Término de medias móviles estacional 2 & 0,900500 & 0,024057 & 0,865235 & 0,017494 \\
\hline Termino de medias móviles MA(4) & & & $-0,300948$ & 0,094904 \\
\hline Termino de medias móviles MA(10) & $-0,219956$ & 0,082594 & $-0,120857$ & 0,079895 \\
\hline Observaciones & 110 & & 125 & \\
\hline Q test (p-valor, retardo 36 ) & $38,365(0,17)$ & & $30,901(0,42)$ & \\
\hline Suma de cuadrados de los residuos & 0,063778 & & 0,098684 & \\
\hline Error estándar de la regresión & 0,024786 & & 0,029167 & \\
\hline Modelo ARIMA & $(2,1,0)(0,0,2) 12$ & & $(2,1,0)(0,0,2) 12$ & \\
\hline
\end{tabular}


Tabla 6

Predicciones del análisis contrafáctico para el gasto farmacéutico del periodo Junio 2012-Agosto 2013

\begin{tabular}{|l|r|r|r|r|}
\hline & Gasto real & Gasto predicho & Intervalo de confianza & \multicolumn{1}{c|}{$\begin{array}{c}\text { Gasto predicho } \\
\text { menos gasto real }\end{array}$} \\
\hline JUN 2012 & 979.492 .540 & 903.891 .726 & $874.701 .819-933.453 .608$ & -75.600 .814 \\
\hline JUL 2012 & 699.143 .499 & 868.602 .210 & $840.805 .104-896.750 .306$ & 169.458 .711 \\
\hline AG 2012 & 713.214 .711 & 880.115 .914 & $849.940 .810-910.699 .585$ & 166.901 .203 \\
\hline SEPT 2012 & 678.853 .084 & 880.899 .179 & $846.249 .815-916.087 .848$ & 202.046 .095 \\
\hline OCT 2012 & 768.785 .967 & 872.561 .688 & $837.952 .390-907.714 .256$ & 103.775 .721 \\
\hline NOV 2012 & 723.057 .428 & 861.214 .097 & $824.830 .937-898.206 .176$ & 138.156 .669 \\
\hline DIC 2012 & 713.151 .960 & 885.173 .864 & $845.732 .226-925.312 .364$ & 172.021 .904 \\
\hline EN 2013 & 787.165 .931 & 883.964 .748 & $843.202 .042-925.473 .256$ & 96.798 .817 \\
\hline FEBR2013 & 718.995 .006 & 887.600 .549 & $845.130 .542-930.877 .377$ & 168.605 .543 \\
\hline MARZ 2013 & 737.495 .091 & 905.605 .078 & $860.797 .355-951.293 .600$ & 168.109 .987 \\
\hline ABR 2013 & 805.341 .015 & 858.971 .231 & $816.151 .045-902.639 .606$ & 53.630 .216 \\
\hline MAY 2013 & 799.346 .997 & 893.885 .047 & $847.868 .065-940.843 .938$ & 94.538 .050 \\
\hline JUN2013 & 755.584 .578 & 892.874 .164 & $835.473 .266-951.749 .777$ & 137.289 .586 \\
\hline JUL 2013 & 794.584 .436 & 857.705 .430 & $801.859 .273-915.005 .209$ & 63.120 .994 \\
\hline AG 2013 & 713.406 .657 & 882.195 .020 & $821.422 .134-944.644 .049$ & 168.788 .363 \\
\hline TOTAL & 11.387 .618 .900 & 13.215 .259 .945 & & 1.827 .641 .045 \\
\hline
\end{tabular}

Figura 4

Análisis contrafáctico de la serie de gasto

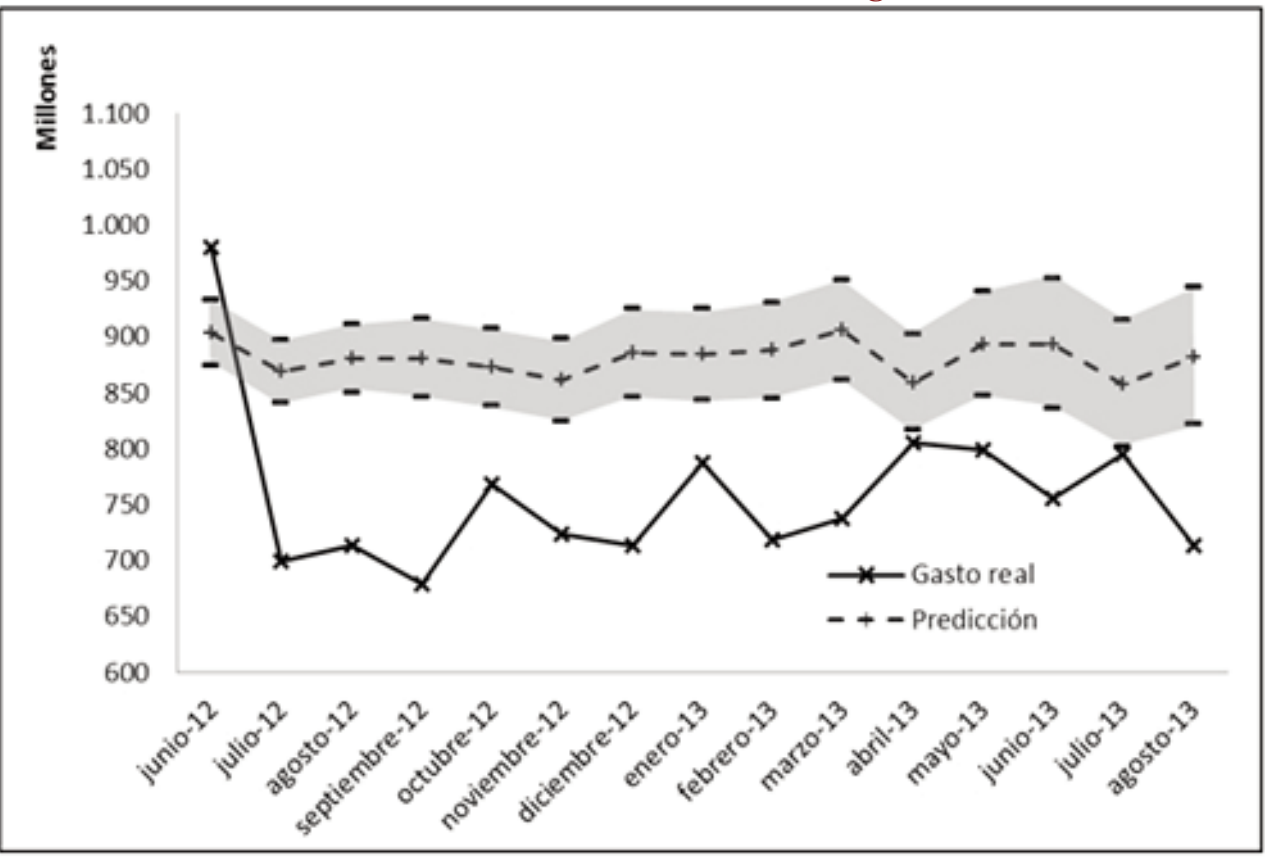


0,056 respectivamente, indicando un descenso en el gasto del 5,6\% y del 5,4\% respectivamente.

El modelo se utilizó para predecir el gasto farmacéutico durante el periodo junio 2012-agosto 2013. Las predicciones se muestran en la tabla 6. El volumen de gasto farmacéutico predicho por el modelo para cada período fue mayor que el volumen real (en el intervalo de 53 a 202 millones de euros) excepto para el mes de junio de 2012, ya que no recogió el efecto del RDL. En la figura 4 se representan las predicciones puntuales para la serie de gasto farmacéutico así como sus correspondientes intervalos de confianza al $80 \%$. Todos los valores reales de la serie de gasto farmacéutico quedaron fuera de estos intervalos, lo cual reflejó que hubo un cambio en la estructura de la serie como consecuencia del RDL. Finalmente, el efecto del RDL fue el de disminuir el volumen de gasto farmacéutico para el período considerado en un 12,83\% (según la fórmula citada).

En el análisis de intervención se estimó el modelo ARIMA $(2,1,0)(0,0,2) 12$ que incluyó las variables DFJUN12, FNOV11 y FJUN10, donde la variable DFJUN12 es la variable en primeras diferencias regulares. Este modelo también exigió la inclusión de dos coeficientes de medias móviles para los retardos 4 y 10 (introducidos en el modelo de forma empírica, a la vista de los correlogramas en las fases de verificación de estimaciones preliminares). Todos los coeficientes estimados fueron estadísticamente significativos al $1 \%$, excepto el coeficiente del término de medias móviles del retardo 10. El coeficiente de la variable DFJUN12 tomó el valor $-0,151215$, interpretándose como que el volumen de gasto disminuyó un 14,03\% debido al RDL (tabla 5).

Figura 5

Serie de gasto con predicciones

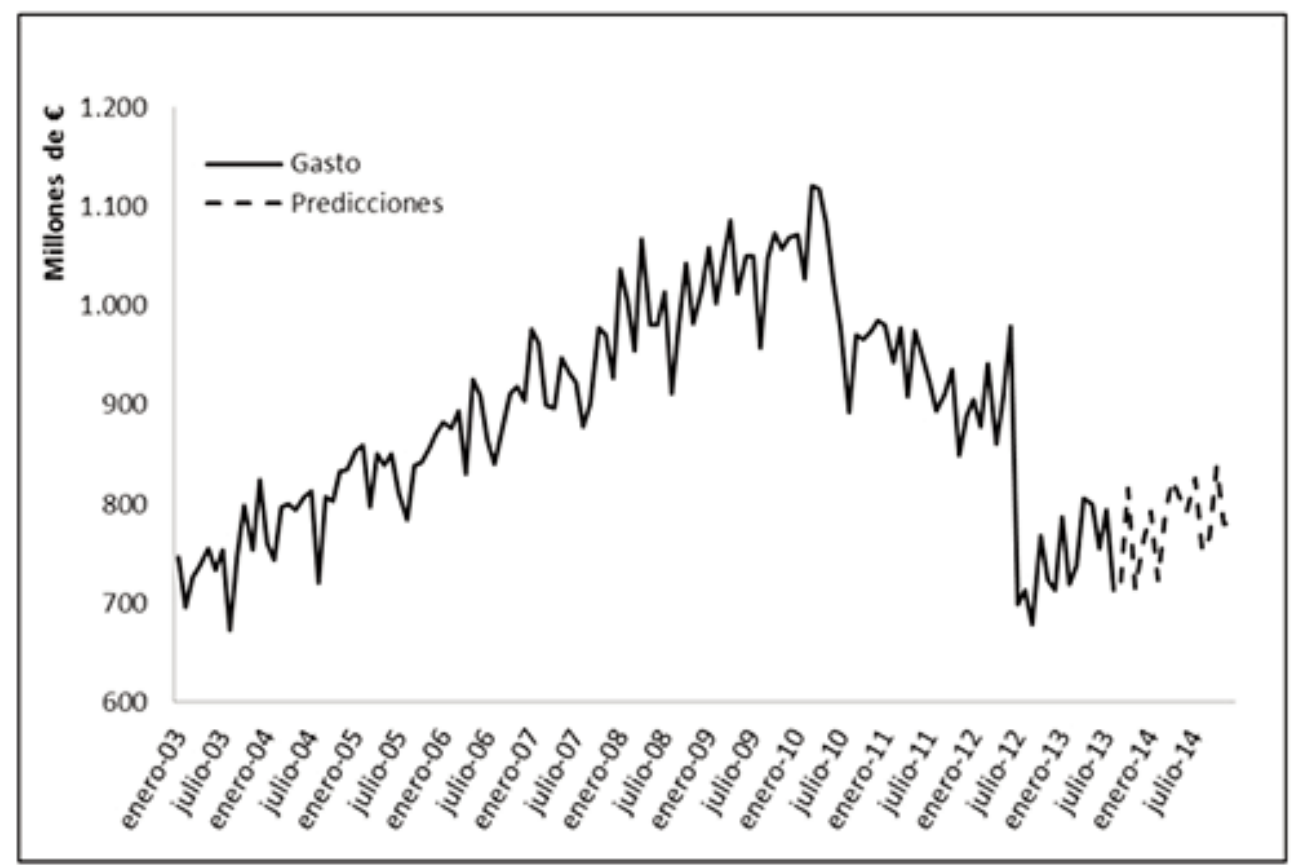


Las predicciones realizadas para el periodo de septiembre de 2013 a diciembre de 2014 se recogen en la tabla 6 y en la figura 5. Las predicciones continuaron con la tendencia creciente que ya apuntaba la serie tras el descenso brusco del verano de 2012.

\section{DISCUSIÓN}

Los modelos ARIMA presentados muestran un error estándar reducido (un 3\% aproximadamente), lo que significa que los intervalos de las predicciones serán también de poca amplitud para un nivel de confianza previamente fijado $(80 \%)$. De esta manera, se podrá analizar con mayor precisión si durante los próximos meses el RDL continúa repercutiendo en las dos variables estudiadas.

Las predicciones de la tabla 4 (junto con las figuras 2 y 5 antes presentadas) muestran un perfil casi estable para el caso de las recetas y una tendencia creciente para el gasto. Esto significaría que el precio medio por receta habría crecido $\mathrm{y}$, dado que hay una menor población asegurada, que se habría producido una sustitución de fármacos de menor precio (quizás los excluidos tras la entrada en vigor del RDL) por otros de precios superiores. En cualquier caso, este particular habría que comprobarlo en un futuro, puesto que no se ha efectuado un análisis específico para la serie temporal del gasto por receta. En este contexto, durante la revisión de la primera redacción del artículo se han conocido las cifras reales del gasto para los meses de septiembre-diciembre de 2013. A excepción del mes de noviembre, las predicciones del modelo son menores en un 1,9\% a los importes reales. En cuanto a la cifra de recetas, el total predicho por el modelo para ese período fue de un 2,4\% menor a la cifra real. Los citados importes reales de gasto se encuentran dentro de los intervalos de confianza calculados, excepto el de noviembre, mientras que para la variable del número de recetas las cifras reales de los cuatro meses se hallan dentro de los intervalos de confianza calculados.
Aunque puede ser algo prematuro establecer esta afirmación porque no ha pasado suficiente tiempo, los efectos del RDL han consistido esencialmente en bajar el nivel del gasto de medicamentos pero la tendencia creciente de antes del verano de 2010 parece que se tiende a retomar, aunque a tasas y niveles absolutos menores. Sin embargo, ha habido un claro cambio en la evolución del número de recetas que ha reducido su nivel y cuya tendencia es casi horizontal mientras que antes era claramente creciente.

Además, cabe resaltar que las cifras del gasto público en medicamentos con prescripción habrán sido menores de lo indicado ya que los pacientes han aumentado su participación por el cambio en los copagos introducido por el RDL. Esto es, los importes citados en las tablas de este artículo corresponden al valor total nominal de la factura farmacéutica (sin descontar lo que pagan los usuarios). En los últimos años, el importe financiado por las administraciones públicas autonómicas era aproximadamente un $94 \%$ del total ${ }^{2}$. En 2012, de acuerdo con los datos del Consejo General de Colegios de Farmacéuticos ${ }^{13}$ hemos podido calcular que esa proporción fue de un 92\% (con solo medio año de aplicación del RDL) por lo que se espera que ese importe sea incluso menor en 2013. Por tanto, la repercusión para la Administración Pública en términos de contención del gasto lograda por el menor número de recetas emitidas y de su correspondiente gasto total se ve también reforzada por esta vía de menores costes en la financiación de los fármacos.

También se han efectuado unos cálculos similares a los desarrollados por el MSSSI para analizar la reducción en el número de recetas y de gasto farmacéutico. De este modo el número total real de recetas emitidas en el periodo de predicción considerado (junio de 2012 hasta agosto de 2013, 15 meses) fue de 1.069.672.880, mientras que en los 15 meses anteriores (marzo de 2011 
hasta mayo de 2012) al comienzo de los efectos de la entrada en vigor del RDL fue de 1.226.090.159. Comparando los dos periodos, el número total de recetas disminuyó un $12,76 \%$, cifra muy cercana al resultado de nuestro modelo ARIMA $(12,18 \%$ en el análisis contrafáctico y $12,75 \%$ en el análisis de intervención).

Algo similar sucede con el gasto farmacéutico total. En el período entre junio de 2012 y agosto de 2013 (15 meses) el gasto se redujo a 11.388 millones de $€$ desde los 13.708 millones de $€$ de los 15 meses anteriores. Comparando en porcentajes ambas cifras, el descenso del gasto fue del $16,92 \%$, que resulta superior al estimado por nuestro modelo ARIMA. Esta mayor divergencia en las reducciones del gasto se puede deber a que el análisis ARIMA incorpora la tendencia decreciente que registraba la serie desde comienzos de 2010 como consecuencia de las fuertes medidas de contención del gasto público. Sin embargo, la comparación del gasto mediante la fórmula con los 15 meses precedentes no captura plenamente esa tendencia decreciente más larga sino que solo tiene en cuenta el repunte experimentado en el segundo semestre de 2011 y primero de 2012 , por lo que la variación relativa parece ser mayor en los 15 últimos meses analizados. Nótese que para el mes de junio de 2012 las predicciones del análisis contrafáctico son inferiores a los valores reales al no incluir el efecto de acumulación producido ante la entrada en vigor de la medida justo en el mes siguiente.

El análisis efectuado mediante los modelos ARIMA tuvo el carácter de univariante, esto es, empleó únicamente los datos de una variable cada mes, o se apoyó en unas variables ficticias para captar algunos elementos deterministas de la variable estudiada. Sin embargo, existen otras variables que durante el período analizado han influido tanto en el número de recetas como en el gasto total en medicamentos que han quedado al margen. Entre ellas, claramente han influido en la dirección de disminuir los consumos de fármacos la menor renta per cápita -ajena a la influencia del RDL-, el hecho de que la población demandante de medicamentos, la población asegurada, desde la entrada en vigor del RDL, se haya reducido en 873.000 con respecto a quienes antes tenían derecho a la atención sanitaria, según ha reconocido el propio Gobierno $^{14}$, la expiración de las patentes de algunos fármacos muy prescritos en atención primaria, la eliminación desde septiembre de 2012 de 400 medicamentos de la financiación pública -también como medida derivada del RDL-, la creciente proporción de medicamentos dispensados en las farmacias hospitalarias respecto a los dispensados en las oficinas de farmacia previamente y la reducción en el número de visitadores médicos, entre otras.

El análisis de intervención capta mediante una variable ficticia la influencia de un fenómeno determinista en la variable objeto de estudio. Sin embargo, en las variables analizadas ya se ha indicado que hubo otras influencias durante este período, algunas propias del RDL y otras independientes pero que también afectaron a las variables. Por esta razón, los resultados obtenidos en forma del valor del coeficiente de la variable ficticia a partir de junio de 2012 habrían captado también otros factores influyentes, cuya cuantificación es muy complicada de determinar. Esto es, la reducción del gasto en farmacia del $14,03 \%$ no es solamente atribuible al RDL.

La variable ficticia diseñada para captar los efectos del RDL asume que el efecto de anticipación en el consumo de junio de 2012 es igual en intensidad y de signo contrario que el efecto del copago de julio en adelante y que el efecto del copago es el mismo mes a mes desde julio de 2012 hasta agosto de 2013, ya que no hay modulación ninguna. Así mismo, no se tuvo en cuenta mediante otra variable ficticia la exclusión de unos 400 medicamentos de la financiación pública que tuvo lugar en septiembre de 2012 como consecuencia del mismo RDL. 
Como se puede deducir de la observación de la figura 3 los últimos 14 resíduos del modelo estimado para la serie de recetas en el análisis de intervención parecen mostrar una cierta estructura -tendrían autocorrelación positiva- que no captó totalmente la variable ficticia diseñada para medir la influencia de dicho RDL. Se efectuaron otras pruebas con diseños alternativos de la intervención (con otras variables ficticias que captaran separadamente los efectos del RDL - una hasta septiembre de 2012 y otra a partir de esa fecha hasta agosto de 2013- que modularan el efecto decreciente de la medida o bien que captaran mediante una variable aislada del tipo impulso el efecto de anticipación de junio de 2012) pero los resultados generales del modelo no mejoraron los descritos en la tabla 2. Similares pruebas se llevaron a cabo para la serie del gasto farmacéutico sin lograr tampoco mejores resultados.

Los modelos ARIMA especificados y estimados tienen componentes estacionales que captan las pautas de utilización de los recursos sanitarios a lo largo del año (menores en verano y mayores en invierno) así como los efectos de posibles normas de frecuencia anual, como las órdenes ministeriales de precios de referencia (tabla 1). Además, han precisado algún parámetro para captar autocorrelaciones trimestrales [MA(3)] halladas en la serie del número de recetas. Este factor trimestral hallado puede deberse a pautas de prescripción de tratamientos crónicos o de contabilización de las variables (efectos de la declaración del IVA,...), pero es un asunto que no se puede afirmar con certeza. En cualquier caso, la inclusión de este parámetro ayuda a disminuir la varianza residual, por lo que se decidió añadirlo en los modelos. En este sentido, en la serie del gasto farmacéutico también se incluyó un componente de $\mathrm{MA}(10)$ cuyo parámetro contribuyó a reducir el error estándar del modelo estimado pero cuya interpretación se desconoce.
Moreno et $\mathrm{al}^{2}$ ya alertaban de que, aparentemente, de la batería de regulaciones aplicadas a los medicamentos en el período 1995-2006, apenas unas pocas habían tenido efecto en la contención del gasto, esencialmente las reducciones de precio y durante solo un corto periodo. Sin embargo, la variable del número total de recetas registraba una tendencia creciente. Ello pudo deberse al gran aumento anual registrado en la población residente (crecimientos de más de 600.000 personas al año por la afluencia de inmigrantes) y en la renta per cápita, que conseguían anular los efectos de buena parte de esas medidas. En nuestro caso, de forma ajena a la influencia causada por el RDL, el descenso registrado en la renta per cápita durante el período analizado habría contribuido a los menores valores de ambas variables.

Los resultados de los modelos ARIMA estimados no son comparables directamente con los obtenidos por Moreno et $\mathrm{al}^{2}$, que siguieron una metodología similar, debido a que las variables objetivo se referían a Cataluña y a que además emplearon los consumos en términos per cápita, datos que no están disponibles de esa forma en las estadísticas del MSSSI. Por otra parte, el período de referencia solo se solapa 4 años con el nuestro, que es más reciente. En nuestro estudio la influencia de anteriores regulaciones a 2006 no resultó relevante ya que la mayor variabilidad de la serie se concentra en épocas más recientes. Así que las comparaciones con el estudio de Moreno et al. no han permitido establecer ninguna pauta común o diferente.

Tras este análisis estadístico cabe destacar que los modelos ARIMA han captado la influencia del RDL así como los de otros fenómenos coincidentes en el tiempo, han cuantificado su impacto en términos absolutos y relativos y han permitido efectuar predicciones a corto plazo hasta diciembre de 2014, de modo que se dispone de 
un instrumento para comprobar en los meses venideros si se mantienen las repercusiones del RDL en cuanto a los objetivos de la reducción del gasto y del número de recetas. Los modelos estimados resultaron ser de calidad estadística en el sentido de que sus errores estándar son inferiores al 3\% y las distintas pruebas estadísticas realizadas para su validación son satisfactorias, por lo que se pueden emplear para hacer un seguimiento futuro de la evolución de estas series (claro está, si no hay otras modificaciones normativas y regulatorias).

Finalmente, en este texto no se ha entrado a valorar en términos de éxito o fracaso las medidas del RDL en cuanto a lograr con un menor consumo de fármacos unos niveles de salud similares a los anteriores a su implantación, lo que constituye una vía de investigación que permanece abierta y es de gran interés. Para ello habría que relacionar las medidas de contención del gasto farmacéutico y sanitario en general con los comportamientos de los pacientes en cuanto a la sustitución de los medicamentos (conociendo sus elasticidades, precio y renta) y las repercusiones de los consumos finales en los posibles cambios registrados en la salud de la población. Para acometer esta tarea serían precisos datos de las familias o de los pacientes acerca de diversas variables, elementos que no se hallan disponibles actualmente.

\section{AGRADECIMIENTOS}

Los autores agradecen a Pedro Gómez del Instituto de Salud Carlos III y a Mónica Ausejo de la Consejería de Sanidad de Madrid sus comentarios y sugerencias a la hora de desarrollar este trabajo. También se agradecen las observaciones y aportaciones para la discusión realizadas por un revisor anónimo, conocedor y experto en esta materia, que han mejorado la versión final del texto.

\section{BIBLIOGRAFÍA}

1. Guillén AM, Cabiedes L. Reforming pharmaceutical policies in the European Union: A "penguin effect"? Int J Health Serv. 2003; 33(1):1-28

2. Moreno-Torres I, Puig-Junoy J, Raya JM. The impact of repeated cost containment policies on pharmaceutical expenditure: Experience in Spain. Eur J Health Econ. 2011; 12(6):563-573

3. Boletín Oficial del Estado. Real Decreto-ley $16 / 2012$, de 20 de abril, de medidas urgentes para garantizar la sostenibilidad del Sistema Nacional de Salud y mejorar la calidad y seguridad de sus prestaciones. BOE núm 98 de 24/04/2012.

4. Ministerio de Sanidad, Servicios Sociales e Igualdad. El gasto farmacéutico descendió en julio casi un $24 \%$ en relación al mismo mes del año anterior, la mayor reducción desde que se elabora el registro. Nota de prensa de 27 de agosto de 2012. [Citado el 25-102013]. Disponible en: www.msssi.gob.es/gabinete/notasPrensa.do? time $=1343772000000$.

5. Ministerio de Sanidad, Servicios Sociales e Igualdad. El ahorro en farmacia alcanza los 2.125 millones de euros a los trece meses de implantarse el nuevo modelo de participación del usuario. [Citado el 25-102013]. Disponible en http://www.msssi.gob.es/gabinete/notasPrensa.do?time $=1375308000000$

6. Box GEP, Jenkins GM, Reinsel GC. Time Series Analysis: Forecasting and Control, 3rd edn. New Jersey: Prentice Hall, Upper Saddle River;1994.

7. Lee Y, Yang M, Huang Y, Liu C, Chen S. Impacts of cost containment strategies on pharmaceutical expenditures of the National Health Insurance in Taiwan, 1996-2003. Pharmacoeconomics. 2006; 24(9):891-902

8. Marshall D, Gough J, Grootendorst P, Buitendyk M, Jaszewski B, Simonyi S, et al. Impact of administrative restrictions on antibiotic use and expenditure in Ontario: Time series analysis. J Health Serv Res Policy. 2006; 11(1):13-20

9. Ong M, Catalano R, Hartig T. A time-series analysis of the effect of increased copayments on the prescription of antidepressants, anxiolytics, and sedatives in Sweden from 1990 to 1999. Clin Ther. 2003; 25(4):1262-1275

10. Pichetti S, Sorasith C, Sermet C. Analysis of the impact of removing mucolytics and expectorants from the list of reimbursable drugs on prescription rates: A time-series analysis for France 1998-2010. Health Policy 2011; 102(2-3):159-169 
11. Box GEP, Tiao GC. Intervention analysis with applications to economic and environmental problems. J. Amer. Stat Assoc. 70, 70-79 (1975)

12. Ministerio de Sanidad, Servicios Sociales e Igualdad. Disponible en: http://www.msssi.gob.es/profesionales/farmacia/datos/home.htm accedido por última vez el 31/10/2013.

13. Consejo General de Colegios de Farmacéuticos. Medicamentos y farmacia en cifras 2012. Disponible en: http://www.portalfarma.com/Profesionales/infoestadistica/Documents/Medicamentos-Farmacia-Cifras-2012.pdf Accedido el 23 de octubre de 2013.

14. Gobierno de España. Plan Nacional de Reformas. Reino de España. 2013. Disponible en http://www.lamoncloa.gob.es/NR/rdonlyres/29B5272BAEC30A47 8CA80F2AB29D675CD4E7/0/PNREspa\%C3\%B1a2 013.pdf Accedido en 24/11/2013. 